\title{
Angina cervical: relato de caso
}

\author{
Geraldo Ávila Reis ${ }^{1}$, Carlos Umberto Pereira ${ }^{2}$, Rodrigo Oliveira Passos ${ }^{3}$, \\ Ana Cristina Lima Santos ${ }^{3}$, José Anísio Santos Júnior ${ }^{3}$ \\ Fundação Beneficente Hospital de Cirurgia, Aracaju, SE, Brasil e Universidade Federal de Sergipe, Aracaju, SE, Brasil.
}

\section{RESUMO}

A angina cervical $(A C)$ é uma síndrome clínica que apresenta diagnóstico diferencial com a angina pectoris, comumente subdiagnosticada. Sua prevalência necessita de dados pela dificuldade diagnóstica como também pela realização de exames e procedimentos desnecessários. A causa mais comum da AC é a compressão das raízes nervosas cervicais. A discectomia cervical tem sido realizada no seu tratamento. Os autores apresentam um caso de angina cervical e discutem seu diagnóstico e conduta.

\section{PALAVRAS-CHAVE}

Descolamento do disco intervertebral, angina pectoris, dor no peito.

\section{ABSTRACT}

Cervical angina: report of case

Cervical angina $(A C)$ is a clinical syndrome that presents differential diagnosis of angina pectoris, commonly misdiagnosed. Its prevalence requires data by diagnostic difficulty and the performance of unnecessary tests. The most common cause of the compression of the cervical nerve roots. The cervical discectomy has been performed in its treatment. The authors present a case of cervical angina and discuss their diagnosis and therapy.

\section{KEYWORDS}

Intervertebral disc displacement, angina pectoris, cesta pain.

1 Médico. Neurocirurgião do Serviço de Neurocirurgia da Fundação Beneficente Hospital de Cirurgia, Aracaju, SE, Brasil.

2 Professor adjunto doutor do Departamento de Medicina da Universidade Federal de Sergipe (UFS). Chefe do Serviço de Neurocirurgia da Fundação Beneficente Hospital de Cirurgia, Aracaju, SE, Brasil.

3 Acadêmicos de Medicina da Universidade Federal de Sergipe, Aracaju, SE, Brasil. 


\section{Introdução}

A pseudoangina é definida como uma dor aguda na região anterior do tórax que simula um quadro de isquemia coronariana, porém de origem não cardíaca. Uma das causas mais comuns dessa condição é a angina

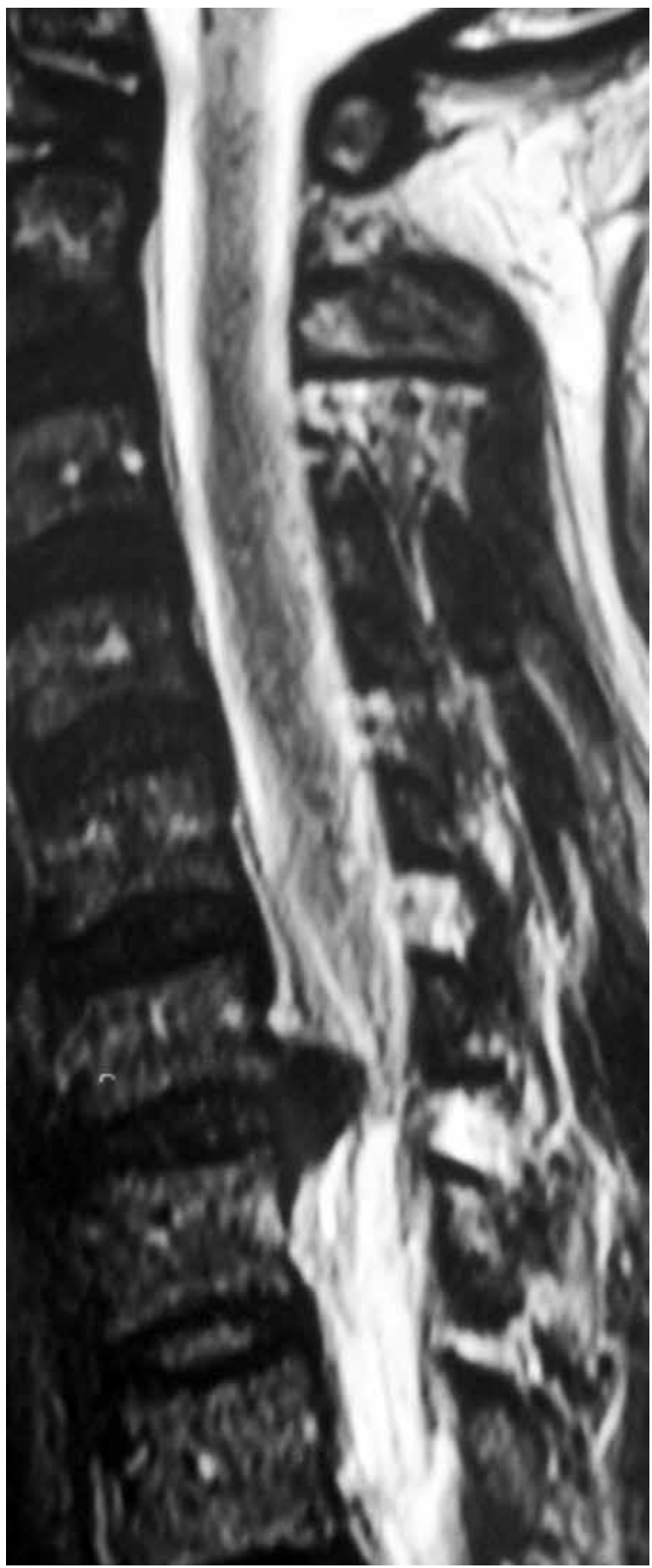

Figura 1 - Ressonância magnética da coluna cervical em corte sagital demonstrando hérnia discal C5-C6. cervical (AC), na qual alterações na coluna cervical manifestam-se com dor precordial e escapular, simulando um quadro de angina pectoris. Geralmente a $\mathrm{AC}$ tem como causa a compressão de raízes nervosas, principalmente no nível de C5, C6 e C7..-6

A prevalência dessa patologia permanece escassa de dados, uma vez que é comumente subdiagnosticada por se tratar de uma síndrome clínica desconhecida. A dificuldade diagnóstica da $\mathrm{AC}$ leva o paciente a ser submetido a uma série de exames e tratamento desnecessários. ${ }^{1,-9}$

Os autores apresentam um caso de AC e discutem o quadro clínico e o tratamento.

\section{Relato do caso}

Paciente de 42 anos de idade, doméstica, deu entrada no serviço de emergência com queixa de dor na região precordial irradiada para membro superior esquerdo (MSE). Refere vários episódios de busca a serviços de emergência, sendo diagnosticada como angina pectoris. Submetida a exames laboratoriais (eletrocardiograma [ECG], troponina, CPK-B e radiografia do tórax) que apresentaram resultados dentro dos padrões de normalidade para idade. Exame físico: bom estado geral, eupneica. Exame neurológico: déficit de força muscular grau 3 e diminuição do reflexo osteotendinoso profundo bicipital $(+/+++)$ em MSE. Exame de ressonância magnética (RM) da coluna cervical (Figuras 1 e 2) demonstrou hérnia discal extrusa no espaço C5-C6. Submetida à discectomia cervical (C5-C6) por via anterior, exérese da hérnia discal, sem intercorrências. Recebeu alta médica hospitalar bem e orientação para retorno ambulatorial.

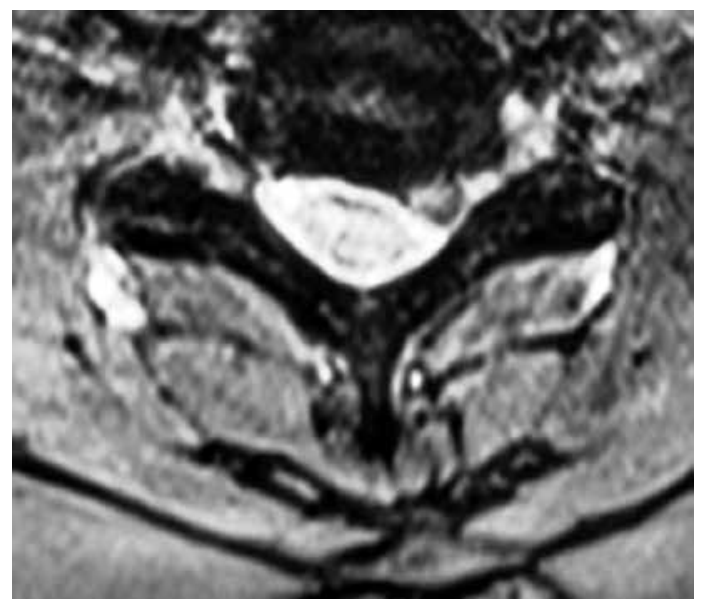

Figura 2 - Ressonância magnética de corte axial apresentando hérnia discal cervical C5-C6 à esquerda. 


\section{Discussão}

As síndromes que cursam com desconforto precordial, porém sem alterações eletrocardiográficas ou arteriográficas, constituem um desafio diagnóstico. Segundo Güler et al., ${ }^{2}$ cerca de $20 \%$ a $30 \%$ dos pacientes com suspeita de angina pectoris têm função coronariana normal e possuem uma origem não cardíaca para o quadro clínico. A raridade de alguns diagnósticos diferenciais, como a AC, faz com que a elucidação diagnóstica seja difícil. ${ }^{10-12}$ Nosso caso confirma essa dificuldade, uma vez que a paciente foi diagnosticada como portadora de angina pectoris em vários serviços de emergência, apesar de os exames complementares serem normais.

A origem da dor na AC ainda não está esclarecida. A teoria mais aceita é a de Brodsky ${ }^{4}$, que afirma tratar-se de uma dor com origem radicular, secundária à compressão de raiz nervosa por hérnia discal, osteófitos ou por estenose de canal cervical. Segundo LaBan et al. ${ }^{13}$, a causa é uma compressão na raiz ventral do neurônio motor, causando uma dor profunda mal definida nas áreas sob inervação da raiz acometida. Para Gonzalez-Darder et al. ${ }^{14}$ seria decorrente da desativação do sistema inibitório descendente. Outras causas são: degeneração discal, síndrome facetária, alterações nos ligamentos longitudinal anterior e posterior. ${ }^{1}$ Em nosso caso, a paciente apresentou hérnia discal extrusa no espaço C5-C6, o que reforça a teoria de Brodsky. ${ }^{4}$

$\mathrm{O}$ reconhecimento da $\mathrm{AC}$ requer uma anamnese detalhada e um exame físico completo. O quadro clínico caracteriza-se por dor na região cervical irradiada para membros superiores (MMSS), déficit de sensibilidade superficial na região paraesternal, cefaleia occipital, dispneia, náuseas, sudorese, diplopia e déficit de força muscular em MMSS. ${ }^{1,4,10}$ Constant ${ }^{15}$ ressalta algumas condições nas quais a angina de origem não cardíaca deve ser uma hipótese diagnóstica, como dor precordial com duração maior que 30 minutos ou menor que cinco segundos, que aumenta com a inspiração ou desaparece em decúbito dorsal, que pode ser desencadeada por movimentação do tronco ou membro superior ou por digitopressão.

Para confirmação diagnóstica, em caso de suspeita de AC, pode-se avaliar o alívio da dor por meio do repouso, uso de colar cervical ou de infiltração local com substâncias anestésicas. Exames de neuroimagens, como tomografia computadorizada e RM, podem confirmar a suspeita de AC. Em nosso caso, a paciente apresentava dor na região cervical irradiada para MSE, déficit de força muscular e diminuição do reflexo bicipital, o que se encontra de acordo com vários autores. ${ }^{1,4,10,15,16}$

O tratamento da AC é cirúrgico. A descompressão cirúrgica pode ser feita por via anterior ou posterior. Em nosso caso, foi submetido à discectomia por via anterior e exérese do disco herniado, fato este realizado por outros autores. . $^{1,3,17}$
A AC é uma síndrome clínica frequentemente subdiagnosticada. A literatura médica necessita de mais dados a respeito da sua prevalência. A falta de conhecimento a respeito da AC tem levado muitos pacientes a serem submetidos a procedimentos de imagens e terapêuticos desnecessários. O diagnóstico correto depende dos sinais e sintomas neurológicos e dos achados de neuroimagens. $\mathrm{O}$ tratamento é cirúrgico e com bom resultado.

\section{Conflitos de interesse}

Os autores declaram não haver conflitos de interesse.

\section{Referências}

1. Nakajima H, Uchida K, Kobayashi S, Kokubo Y, Yayama T, Sato R, et al. Cervical angina: a seemingly still neglected symptom of cervical spine disorder? Spinal Cord. 2006;44(8):509-13.

2. Güler N, Bilge M, Eryonucu B, Cirak B. Acute ECG changes and chest pain induced by neck motion in patients with cervical hernia--a case report. Angiology. 2000;51(10): 861-5.

3. Nakae Y, Johkura K, Kudo Y, Kuroiwa Y. Spinal cord infarction with cervical angina. J Neurol Sci. 2013;324(12):195-6.

4. Brodsky AE. Cervical angina. A correlative study with emphasis on the use of coronary arteriography. Spine (Phila Pa 1976). 1985;10(8):699-709.

5. Jacobs B. Cervical angina. N Y State J Med. 1990;90(1): 8-11.

6. Sudo H, Goto R. Cervical angina because of ossification of the posterior longitudinal ligament. Spine J. 2012;12(2):169.

7. Christensen HW, Vach W, Gichangi A, Manniche C, Haghfelt T, Høilund-Carlsen PF. Cervicothoracic angina identified by case history and palpation findings in patients with stable angina pectoris. J Manipulative Physiol Ther. 2005;28(5):303-11.

8. Passmore SR, Dunn AS. Positive patient outcome after spinal manipulation in a case of cervical angina. Man Ther. 2009;14(6):702-5.

9. Ito $\mathrm{Y}$, Tanaka N, Fujimoto $\mathrm{Y}$, Yasunaga $\mathrm{Y}$, Ishida O, Ochi M. Cervical angina caused by atlantoaxial instability. J Spinal Disord Tech. 2004;17(5):462-5.

10. Lenfant $\mathrm{C}$. Chest pain of cardiac and noncardiac origin. Metabolism. 2010;59(Suppl 1):41-6.

11. Mathers JJ. Differential diagnosis of a patient referred to physical therapy with neck pain: a case study of a patient with an atypical presentation ofangina. J Man Manip Ther. 2012;20(4):214-8.

12. Cook C, Hegedus E. Diagnostic utility of clinical tests for spinal dysfunction. Man Ther. 2011;16(1):21-5.

13. LaBan MM, Meerschaert JR, Taylor RS. Breast pain: a symptom of cervical radiculopathy. Arch Phys Med Rehabil. 1979;60(7):315-7. 
14. Gonzalez-Darder JM, Gonzalez-Martinez V, Canela-Moya P. Cervical spinal cord stimulation in the treatment of severe angina pectoris. Neurosurg Quart. 1998;8:16-23.

15. Constant J. The diagnosis of nonanginal chest pain. Keio J Med. 1990;39(3):187-92.

16. Wells P. Cervical angina. Am Fam Physician. 1997;55(6):2262-4.

17. Furlan JC, Fehlings MG. Cardiovascular complications after acute spinal cord injury: pathophysiology, diagnosis, and management. Neurosurg Focus. 2008;25(5):E13.
Endereço para correspondência

Carlos Umberto Pereira

Av. Augusto Maynard, 245/404, Bairro São José

49015-380 - Aracaju, Se, Brasil

E-mail: umberto@infonet.com.b 